\title{
Microwave Assisted Fast Synthesis of CuO Nanoflakes: Catalytic Application in the Synthesis of 1,4-Dihydropyridine
}

\author{
S.D. BAJAJ ${ }^{a}$, P.V. TeKAdE ${ }^{a, *}$, G.V. LAKhotiya ${ }^{b}$ And P.G. BorkAR ${ }^{a}$ \\ ${ }^{a}$ Department of Chemistry, Jankidevi Bajaj College of Science, Jamnalal Bajaj Marg, Civil Lines, \\ Wardha-442001, India \\ ${ }^{b}$ Department of Physics, Jankidevi Bajaj College of Science, Jamnalal Bajaj Marg, Civil Lines, \\ Wardha-442001, India
}

(Received 13 October 2016; in final form 12 April 2017)

\begin{abstract}
$\mathrm{CuO}$ nanoflakes were successfully synthesized by microwave irradiation, using copper(II) sulphate and sodium hydroxide $(\mathrm{NaOH})$ as the starting materials and ethanol as the solvent. The $\mathrm{CuO}$ nanoflakes were characterized by using techniques such as X-ray powder diffraction, field emission scanning electron microscopy, and UV-visible absorption spectroscopy, Fourier transform infra-red spectroscopy. The synthesized $\mathrm{CuO}$ nanoflakes were found to have morphology like nanoflakes with narrow size distribution and high purity. Moreover, the synthesized CuO nanoflakes were used as an efficient catalyst for synthesis of a series of dihydropyridine derivatives. Optimization studies with different catalysts and solvents reveal that $\mathrm{CuO}$ nanoparticle in the water/ethanol mixture is efficient catalyst/solvent system for the synthesis of dihydropyridine derivative.
\end{abstract}

DOI: 10.12693/APhysPolA.132.1294

PACS/topics: microwave irradiation, $\mathrm{CuO}$ nanoflakes, catalyst, dihydropyridine

\section{Introduction}

In recent years, application of nanoparticles as a catalyst in organic synthesis gained considerable attention because they are reusable and removable from the reaction medium by simple filtration. Moreover, nanocatalysts reported to have high efficiency owing to their large surface to volume ratio, which increases their catalytic activity. Likewise, the supported magnetic nanocatalysts are of considerable interest in organic synthesis because they are feasible alternatives to conventional catalysts, readily obtainable, easily separable by an external magnet and high degree of chemical stability in most of the organic and inorganic solvents [1-10]. The dihydropyridine derivatives are one of the important heterocyclic ring systems because of their extensive pharmacological properties and they are also analogues of NADH coenzymes. Moreover, the dihydropyridyl compounds possesses a wide range of biological activity and medicinal properties e.g. smooth muscle selective, antihistamines, cardioselective. The dihydropyridines is a topic of interest for study because they have fascinating heterocyclic nucleus with multiple reaction centers [11, 12].

Furthermore, cytotoxic activity of 4-substituted phenyl dihydropyridines against human oral squamous carcinoma is an important aspect. The well known dihydropyridine compounds such as nifedipine, nitrendipine and nimodipine $[13,14]$ were reported as commercially useful molecules which are calcium channel blockers. Consequently, a variety of potential drugs are available for the treatment of congestive heart failure $[15,16]$ and neuroprotective agent [17] which possesses dihydropyridine

*corresponding author; e-mail: pradiptekade@gmail.com nucleus. In addition, various medicinal applications viz. antihypertensive, hepatoprotective, bronchodilators, geroprotective, antiatherosclerotic, antitumour, antiplatelet, antimutagenic, antidiabetic, and aggregation activity were shown by some of the dihydropyridine derivatives [18-21]. These compounds were also found as potential calcium channel blockers in medicinal and bioorganic chemistry [22] and NADH models [23], respectively. Moreover, the 1,4-dihydropyridines also acts as a flexible intermediate in the synthesis of natural products owing to their higher reactivity [24].

Consequently, various synthetic methods were developed owing to a wide range of biological medicinal and pharmacological importance of dihydropyridine derivatives. Some of the synthetic methods are use of microwave irradiation technique $[25,26]$, refluxing at high temperature [27-31], use of ionic liquid [32], use of catalysts like $\mathrm{SiO}_{2} / \mathrm{HClO}_{4}$ [33], $\mathrm{SiO}_{2} / \mathrm{NaHSO}_{4}$ [34], I [35], bakers' yeast [36], metal triflates [37], tetrabutylammonium hydrogen sulfate [38], organocatalysts [39], PTSA [40], iron trifluoroacetate [41], TMSCl [42] and Ni nanoparticles [43].

Literature survey reveals that various synthetic methodology for synthesis of 1,4-dihydropyridine derivatives possesses some disadvantages such as costly reagents, lengthy work-up procedures, moisture sensitiveness, toxic and harsh reaction conditions. Therefore, development of fast and efficient methodology with an efficient, green solvent/catalyst system for the synthesis of 1,4-dihydropyridine is an important challenge for organic chemists.

Therefore, it is a topic of interest to synthesize nanoparticles with high surface area and to focus their catalytic application in organic synthesis [44-48]. Therefore, we reported herein $\mathrm{CuO}$ nanoflakes as a efficient 
catalyst for the synthesis of 1,4-dihydropyridine derivatives through one-pot four-component Hantzsch condensation reaction using aromatic aldehyde, ethyl acetoacetate/methyl acetoacetate and ammonium acetate in ethanol as solvent, in excellent yield utilizing microwave assisted technology. The novelty of this proposed method lies in the use of ethanol as a green, less toxic solvent, low-cost catalyst (synthesized in laboratory with readily available staring materials) and simple synthetic methodology, mild reaction conditions, high yields, simple workup, easy filtration and reusability of catalyst.

\section{Experimental}

\subsection{General}

All solvents, chemicals and reagents procured from Merck and Sigma Aldrich. Melting points were determined on Digital Melting Point apparatus and are uncorrected. The field emission scanning electron microscopy (FESEM) images were obtained on a Seron AIS 2100. The Fourier transform infrared (FT-IR) spectra were recorded on a Bruker alpha FTIR spectrometer and UVvis spectroscopy was studied on Shimadzu 1800 setup at J.B. Science College, Wardha. The ${ }^{1} \mathrm{H}$ NMR spectra were recorded on Bruker DRX- 300 Avance spectrometer at $300.13 \mathrm{MHz}$ at SAIF, Chandigarh, Punjab University, and Punjab. The elemental analyses were done with an Elemental Analyser system at SAIF, Chandigarh, Punjab University, and Punjab. All the reagents used in this synthesis are of analytical grade and used without further purification. The X-ray diffraction (XRD), FESEM analyses were carried out at SAIF, IIT Bombay. BET surface area analysis was studied at Material Science Center, IIT Kharagpur.

\section{2. $\mathrm{CuO}$ nanomaterial preparation}

$25 \mathrm{ml}$ ethanolic solution of $0.5 \mathrm{M} \mathrm{CuSO}_{4} \cdot 5 \mathrm{H}_{2} \mathrm{O}$ (copper(II) sulfate 5-hydrate, 99.95\%) stirred for 5 min on magnetic stirrer and $25 \mathrm{ml}$ ethanolic solution of $0.5 \mathrm{M}$ $\mathrm{NaOH}$ added dropwise to the ethanolic solution of copper sulphate with vigorous stirring for about $15 \mathrm{~min}$. The resulting mixture placed in the scientific microwave oven with the specification of $650 \mathrm{~W}$ for about $10 \mathrm{~min}$. During the reflux the change in color of the solution varies from sky blue to colorless and then slowly turns to brick red. This indicates the formation of $\mathrm{CuO}$ nanomaterial. The solution were cooled to room temperature and the obtained precipitate was centrifuged, washed first with distilled water and then with absolute ethanol and with acetone in sequence and dried in oven at $100^{\circ} \mathrm{C}$ for $3 \mathrm{~h}$. The final product was collected and used for characterization.

\subsection{Synthesis of diethyl 2,6-dimethyl-4-(4-nitrophenyl)- 1,4-dihydropyridine-3,5-dicarboxylate}

A mixture of an aromatic aldehyde (1 mmol), ethyl acetoacetate $(2 \mathrm{mmol})$, and ammonium acetate
$(1.3 \mathrm{mmol})$ and copper oxide nanoflakes $(\mathrm{CuO})$, vigorously agitated in water $(2 \mathrm{~mL})$ at $70^{\circ} \mathrm{C}$ for the stipulated period of time, till the completion of the reaction (checked by TLC). After completion of the reaction, few drops of ethanol were added to the reaction mixture (to assist granulation of the products). The catalyst, being insoluble in ethanol/water, was separated by centrifugation. The catalyst was removed by filtration and the filtrate was added to crushed ice. A solid product was obtained, which was filtered, washed with water, and crystallized from aqueous ethanol, if needed (Fig. 1).

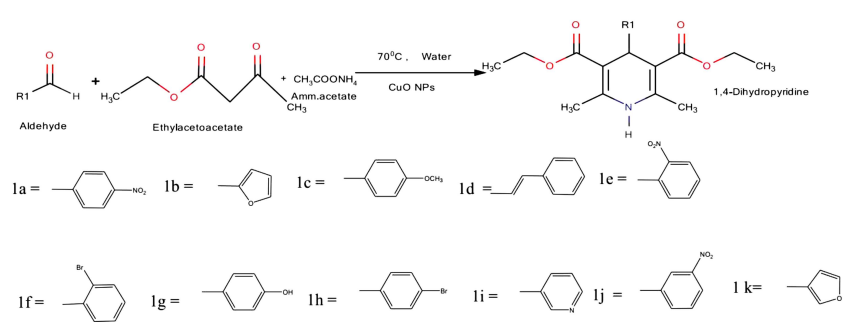

Fig. 1. Scheme of one pot four component synthesis of 1,4-dihydropyridine.

\subsection{Recycling of catalyst}

To examine the reusability, the catalyst was recovered by filtration from the reaction mixture after dilution with ethanol, washed with methanol, and reused as such for subsequent experiments (up to three cycles) under similar reaction conditions. The observed fact that yields of the product remained comparable in these experiments established (Table I) the recyclability and reusability of the catalyst without any significant loss of activity.

Recyclability of catalyst.

TABLE I

\begin{tabular}{c|c}
\hline \hline Number of cycle & \% Yield \\
\hline 1st & 93 \\
2nd & 90 \\
3rd & 89
\end{tabular}

\subsection{Optimization studies of synthesis of 1,4-dihydropyridine}

In the present work, the reaction conditions optimized with respect to the catalyst, solvent and reactants for carbon-carbon $(\mathrm{C}-\mathrm{C})$ and carbon-heteroatom $(\mathrm{C}-\mathrm{N})$ bond formation. The physical characterization is given in Table II.

In continuation of this research, the reaction conditions were optimized on the basis of the catalyst, solvent and reactants for carbon-carbon and carbon-heteroatom bond formation. To test the efficiency of the catalytic activity, we chose to focus our initial studies on the cyclization reaction of aldehydes, ammonium actetate and ethylacetoacetate in the presence of different catalysts such as $\mathrm{Al}_{2} \mathrm{O}_{3}, \mathrm{TiO}_{2} \mathrm{SiO}_{2}, \mathrm{CuO}$ and also in the presence of regular $\mathrm{CuO}$ (purchased from Sigma Aldrich). 
TABLE II

Physical characterization of the synthesized compounds.

\begin{tabular}{|c|c|c|c|c|c|c|c|c|}
\hline$\overline{\mathrm{SN}}$ & Code & Structure & M.F. & M.W. & $\begin{array}{l}\text { Time } \\
{[\mathrm{min}]}\end{array}$ & \begin{tabular}{|l|} 
M.P \\
{$\left[{ }^{\circ} \mathrm{C}\right]$}
\end{tabular} & $\begin{array}{c}\text { Yield } \\
{[\%]} \\
\%]\end{array}$ & $R_{f}$ \\
\hline 1 & $1 \mathrm{a}$ & & $\mathrm{C}_{19} \mathrm{H}_{22} \mathrm{~N}_{2} \mathrm{O}_{6}$ & 374.38 & 120 & $\begin{array}{c}169 \\
\div \\
171\end{array}$ & 93 & 0.54 \\
\hline 2 & $1 \mathrm{~b}$ & & $\mathrm{C}_{17} \mathrm{H}_{21} \mathrm{NO}_{5}$ & 319.35 & 115 & $\begin{array}{c}165 \\
\div \\
168\end{array}$ & 90 & 0.57 \\
\hline 3 & $1 \mathrm{c}$ & & $\mathrm{C}_{20} \mathrm{H}_{25} \mathrm{NO}_{5}$ & 359.41 & 100 & $\begin{array}{c}160 \\
\div \\
162\end{array}$ & 85 & 0.65 \\
\hline 4 & $1 \mathrm{~d}$ & & $\mathrm{C}_{21} \mathrm{H}_{25} \mathrm{NO}_{4}$ & 355.43 & 105 & $\begin{array}{c}223 \\
\div \\
225\end{array}$ & 85 & 0.70 \\
\hline 5 & $1 \mathrm{e}$ & & $\mathrm{C}_{19} \mathrm{H}_{22} \mathrm{~N}_{2} \mathrm{O}_{6}$ & 374.38 & 105 & $\begin{array}{c}172 \\
\div \\
174\end{array}$ & 75 & 0.65 \\
\hline 6 & 1f & & $\mathrm{C}_{19} \mathrm{H}_{22} \mathrm{BrNO}_{4}$ & 408.29 & 95 & $\mid \begin{array}{c}210 \\
\div \\
213\end{array}$ & 65 & 0.65 \\
\hline 7 & $1 \mathrm{~g}$ & & $\mathrm{C}_{19} \mathrm{H}_{23} \mathrm{NO}_{5}$ & 345.39 & 80 & $\begin{array}{c}241 \\
\div \\
242\end{array}$ & 93 & 0.60 \\
\hline 8 & $1 \mathrm{~h}$ & & $\mathrm{C}_{19} \mathrm{H}_{22} \mathrm{BrNO}_{4}$ & 408.29 & 95 & $\begin{array}{c}210 \\
\div \\
213\end{array}$ & 65 & 0.65 \\
\hline 9 & $1 \mathrm{i}$ & & $\mathrm{C}_{18} \mathrm{H}_{22} \mathrm{~N}_{2} \mathrm{O}_{4}$ & 330.38 & 60 & $\begin{array}{c}211 \\
\div \\
212\end{array}$ & 90 & 0.60 \\
\hline 10 & $1 \mathrm{j}$ & & $\mathrm{C}_{19} \mathrm{H}_{22} \mathrm{~N}_{2} \mathrm{O}_{6}$ & 374.39 & 102 & $\begin{array}{c}223 \\
\div \\
225\end{array}$ & 88 & 0.70 \\
\hline 11 & $1 \mathrm{k}$ & & $\mathrm{C}_{17} \mathrm{H}_{21} \mathrm{NO}_{5}$ & 319.35 & 115 & $\begin{array}{c}165 \\
\div \\
168\end{array}$ & 90 & 0.57 \\
\hline
\end{tabular}

The significant results of Table III corresponds to the reactivity of catalytic nanoparticles determined by the energy of surface atoms on surface of nanoparticle.

For the optimization of the reaction conditions, we proceeded the model reaction using $\mathrm{CuO}$ nanoparticles in various solvents.
TABLE III

One-pot synthesis of diethyl 2,6-dimethyl-4-(4nitrophenyl)-1,4-dihydropyridine-3,5-dicarboxylate in various catalysts.

\begin{tabular}{c|c|c|c}
\hline \hline Entry & Catalyst & Time $[\mathrm{min}]$ & Yields $^{a}[\%]$ \\
\hline 1 & $\mathrm{SiO}_{2}$ & 100 & 35 \\
2 & $\mathrm{Al}_{2} \mathrm{O}_{3}$ & 120 & 50 \\
3 & $\mathrm{TiO}_{2}$ & 140 & 70 \\
4 & $\mathrm{CuO}$ & 160 & 60 \\
5 & $\mathrm{CuONps}$ & 120 & $85-90$
\end{tabular}

${ }^{a}$ isolated yields

TABLE IV

One-pot synthesis of diethyl 2,6-dimethyl-4-(4nitrophenyl)-1,4-dihydropyridine-3,5-dicarboxylate in various solvents ${ }^{a}$.

\begin{tabular}{c|c|c|c}
\hline \hline Entry & Solvent & Time [min] & Yields [\%] $^{b}$ \\
\hline 1 & toluene & 240 & 35 \\
2 & acetonitrile & 180 & 50 \\
3 & ethanol & 120 & 70 \\
4 & ethanol/water & 140 & $80-90$
\end{tabular}

${ }^{a}$ reflux conditions, ${ }^{b}$ isolated yields.

The results in Table IV show that a mixture of water/ethanol is the most efficient solvent for this multicomponent reaction. This is not surprising, insight of the fact that the hydrogen bonding between water/ethanol and the substrate can promote the nucleophilic attack of the reactants.

\section{Results and discussion}

The $\mathrm{CuO}$ nanocatalyst was synthesized by a modified precipitation method in which the reaction was carried out under scientific microwave oven using an ethanol/water mixture (30:70) as a green medium. Then, it was characterized by powder X-ray diffraction (PXRD), scanning electron microscopy (SEM), and FTIR spectral analysis. The SEM analysis was done to study particle size and the $\mathrm{CuO}$ nanoparticle morphology was identified based on the analysis of SEM images.

\subsection{Characterization of $\mathrm{CuO}$ nanoparticles}

\subsection{1. $X R D$}

The structural property of as-synthesized $\mathrm{CuO}$ nanomaterial is analyzed by the powder XRD. Figure 2 illustrates the XRD patterns of as-synthesized $\mathrm{CuO}$ nanostructures. The XRD pattern is the replica of the JCPDS pattern with reference code 00-044-0706. No characteristic peaks of impure phases, such as $\mathrm{Cu}(\mathrm{OH})_{2}$ or $\mathrm{Cu}_{2} \mathrm{O}$ were detected. This indicates the formation of single phase pure monoclinic $\mathrm{CuO}$ nanomaterial.

\subsubsection{FESEM}

Figure 3 shows the morphology and size of the synthesized nanomaterial investigated by FESEM. FESEM images of as synthesized $\mathrm{CuO}$ reveal the growth of nanoparticles from the nucleating center whose maximum diameter is around $400 \mathrm{~nm}$ (Fig. 3). Morphology and size of the 


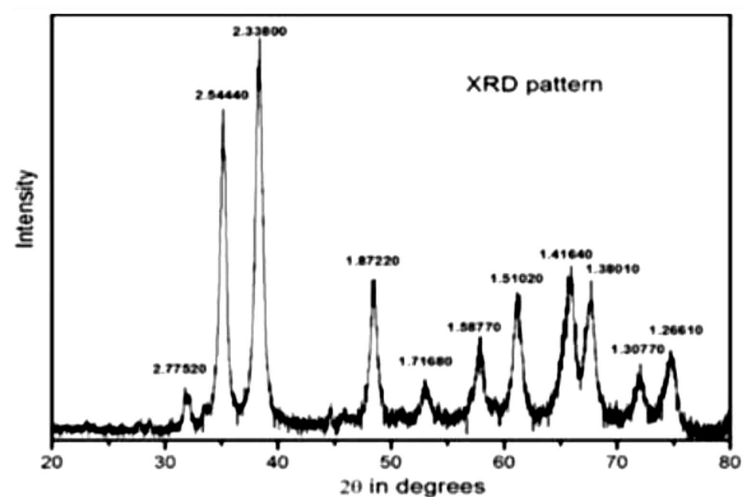

Fig. 2. Powder X-ray diffraction pattern of as synthesized $\mathrm{CuO}$ nanomaterial.

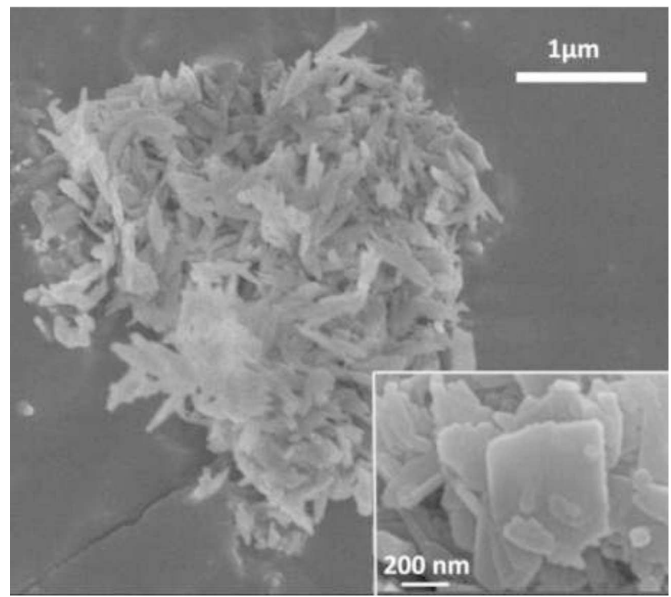

Fig. 3. FESEM image for $\mathrm{CuO}$ nanoflakes.

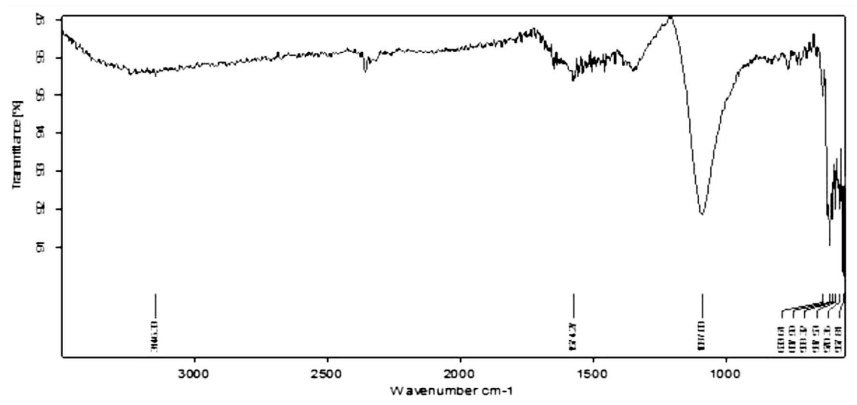

Fig. 4. FTIR spectrum of $\mathrm{CuO}$ nanoflakes.

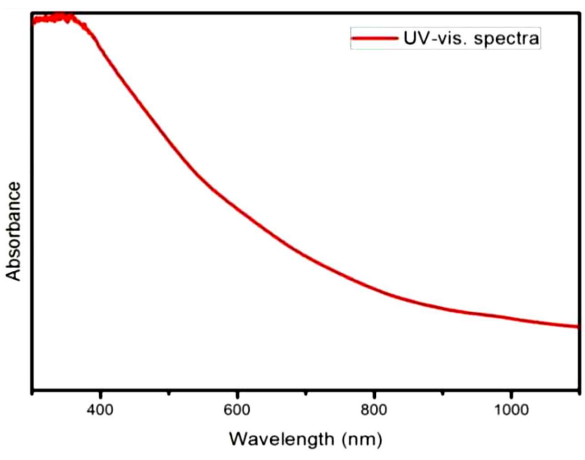

Fig. 5. UV-vis spectra of $\mathrm{CuO}$ nanoflakes.

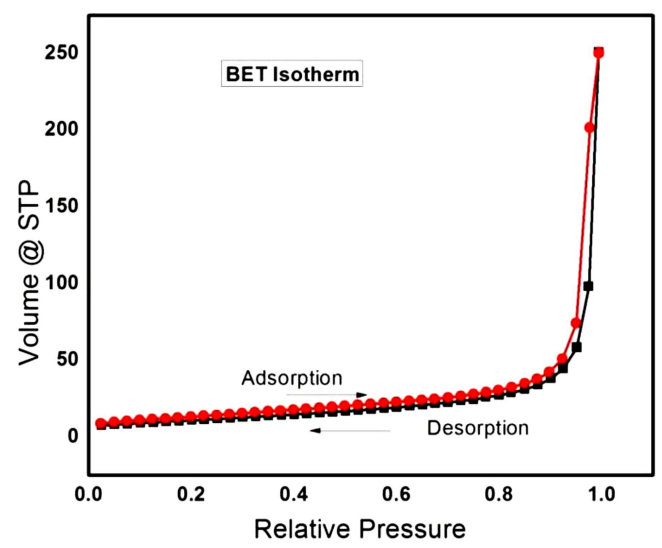

Fig. 6. BET isotherm of as synthesized $\mathrm{CuO}$ nanoflakes.

nanoparticles can be confirmed from the inset containing magnified image of the same.

\subsubsection{FTIR}

The synthesized nanostructure subjected to FTIR investigation (Fig. 4). The spectrum exhibited several strong bands below $700 \mathrm{~cm}^{-1}$ i.e. at $633,607,598,587$, $570,557 \mathrm{~cm}^{-1}(\mathrm{Cu}-\mathrm{O}$ link stretch and $\mathrm{O}-\mathrm{Cu}-\mathrm{O}$ asymmetric vibrations) which confirmed the formation of monoclinic $\mathrm{CuO}$ phase. The characteristic band at $607 \mathrm{~cm}^{-1}$ is due to $\mathrm{Cu}-\mathrm{O}$ stretching.

\subsubsection{UV-vis spectroscopy}

Figure 5 depicts the UV-vis spectra of as synthesized $\mathrm{CuO}$ nanomaterial which shows the wide absorption in visible region which is in well agreement with the $\mathrm{CuO}$ nanostructures.

\subsubsection{BET surface area}

The effective Brunauer-Emmett-Teller (BET) surface area of the as-synthesized samples was measured using a Quantachrome ChemBET TPR/TPD analyzer. Figure 6 shows the isotherm of $\mathrm{N}_{2}$ adsorption of the nanoparticles. The effective BET specific surface area of as synthesized nanoparticles is found to be $17.09 \mathrm{~m}^{2} / \mathrm{g}$.

\subsection{Spectroscopic data of synthesized compounds}

IR, ${ }^{1} \mathrm{H}$ NMR, ${ }^{13} \mathrm{C}$ NMR and mass spectral data of compounds 1a-1f are given below. The spectral data are in good correlation to the suggested structure of the compounds.

1. Diethyl 2,6-dimethyl-4-(4-nitrophenyl)-1,4dihydropyridine-3,5-dicarboxylate (1a):

\% Yield: 85 ; m.p. $169-171^{\circ} \mathrm{C}$.

IR, $\nu_{\max } / \mathrm{cm}^{-1}: 3342(\mathrm{NH}), 1703(\mathrm{CO}), 1643(\mathrm{CO})$, $1523(=\mathrm{C}-\mathrm{H}$ and ring $\mathrm{C}=\mathrm{C}), 1485(\mathrm{C}=\mathrm{C}), 1369$ (sym. $\mathrm{CH}_{3}$ in $\left.\mathrm{O}-\mathrm{CH}_{2}-\mathrm{CH}_{3}\right), 1345(\mathrm{CN}), 1299-1258$ (C-O-C). ${ }^{1} \mathrm{H} \mathrm{NMR}\left(500.1 \mathrm{MHz}, \mathrm{CDCl}_{3}\right), \delta: 1.23$ (3 H,Me), 2.40 $\left(3 \mathrm{H}, \mathrm{CH}_{3}\right), 4.09\left(2 \mathrm{H}, \mathrm{CH}_{2}\right), 5.09(1 \mathrm{H}, \mathrm{CH}), 5.80$ (1 $\mathrm{H}, \mathrm{NH}), 7.27-8.13(4 \mathrm{H}, \mathrm{Ar}-\mathrm{H}) .{ }^{13} \mathrm{C} \mathrm{NMR}(125.8 \mathrm{MHz}$, $\left.\mathrm{CDCl}_{3}\right), \delta: 14.88\left(\mathrm{CH}_{3}\right), 18.66\left(\mathrm{CH}_{3}\right), 59.35\left(\mathrm{CH}_{2}\right)$, 
$167.56(\mathrm{C}=\mathrm{O}), 102-154(\mathrm{Ar}-\mathrm{C})$. LCMS $(\mathrm{m} / \mathrm{z})$ : calculated $\left(\mathrm{M}^{+}+\mathrm{Na}\right)$ : 397, found 397.18.

2. Diethyl 4-(furan-2-yl)-2,6-dimethyl-1,4-dihydropyridine-3,5-dicarboxylate $(1 \mathrm{~b})$ :

$\%$ Yield, 90 ; m.p. $165-168^{\circ} \mathrm{C}$.

$\mathrm{IR}, \nu_{\max } / \mathrm{cm}^{-1}: 3334(\mathrm{NH}), 2978(\mathrm{C}-\mathrm{H}), 1686(\mathrm{C}=\mathrm{O})$, $1639(\mathrm{C}=\mathrm{O}), 1529(=\mathrm{C}-\mathrm{H}$ and $\mathrm{C}=\mathrm{C}), 1487(\mathrm{C}=\mathrm{C}$ in furan), $1371\left(\mathrm{CH}_{3}\right.$ in $\left.\mathrm{O}-\mathrm{CH}_{2}-\mathrm{CH}_{3}\right), 1325\left(\mathrm{C}-\mathrm{N}\right.$ in $\left.\mathrm{C}_{A r}-\mathrm{N}\right)$, $1296(\mathrm{C}-\mathrm{O}-\mathrm{C}) .{ }^{1} \mathrm{H}$ NMR $\left(500.1 \mathrm{MHz}, \mathrm{CDCl}_{3}\right), \delta: 1.26$ $\left(3 \mathrm{H}, \mathrm{CH}_{3}\right), 2.33\left(2 \mathrm{H}, \mathrm{CH}_{2}\right), 4.14\left(3 \mathrm{H}, \mathrm{CH}_{3}\right), 5.20(1 \mathrm{H}$, $\mathrm{CH}), 5.81(1 \mathrm{H}, \mathrm{NH}), 5.94-7.27(3 \mathrm{H}, \mathrm{Ar}-\mathrm{H})$.

3. Diethyl 4-(4-methoxphenyl)-2,6-dimethyl-1,4dihydropyridine-3,5-dicarboxylate (1c):

$\%$ Yield, 85 ; m.p. $160-162{ }^{\circ} \mathrm{C}$

IR $\nu_{\max } / \mathrm{cm}^{-1}: 3324(\mathrm{~N}-\mathrm{H}), 2974(\mathrm{C}-\mathrm{H}), 1696(\mathrm{C}=\mathrm{O})$, $1649(\mathrm{C}=\mathrm{O}), 1529(=\mathrm{C}-\mathrm{H}$ and ring $\mathrm{C}=\mathrm{C}), 1487(\mathrm{C}=\mathrm{C})$, $1371\left(\mathrm{CH}_{3}\right.$ in $\left.\mathrm{O}-\mathrm{CH}_{2}-\mathrm{CH}_{3}\right), 1326\left(\mathrm{C}-\mathrm{N}\right.$ in $\left.\mathrm{C}_{A r}-\mathrm{N}\right), 1295$ $(\mathrm{C}-\mathrm{O}-\mathrm{C}) .{ }^{1} \mathrm{H}$ NMR $\left(500.1 \mathrm{MHz}, \mathrm{CDCl}_{3}\right), \delta: 0.88(3 \mathrm{H}$, $\left.\mathrm{CH}_{3}\right), 1.23\left(2 \mathrm{H}, \mathrm{CH}_{2}\right), 2.33\left(3 \mathrm{H}, \mathrm{CH}_{3}\right), 3.90(1 \mathrm{H}, \mathrm{CH})$, $4.07(1 \mathrm{H}, \mathrm{NH}), 6.74-7.26(4 \mathrm{H}, \mathrm{Ar}-\mathrm{H})$.

4. Diethyl-2,6-dimethyl-4-(2-phenylethylene)-1,4dihydropyridine-3,5-dicarboxylate (1d):

$\%$ Yield, 85 ; m.p. $223-225^{\circ} \mathrm{C}$.

IR $\nu_{\max } / \mathrm{cm}^{-1}: 3334(\mathrm{~N}-\mathrm{H}), 2978(\mathrm{C}-\mathrm{H}), 1686(\mathrm{C}=\mathrm{O})$, $1639(\mathrm{C}=\mathrm{O}), 1487(\mathrm{C}=\mathrm{C}), 1214(\mathrm{C}-\mathrm{O}-\mathrm{C})$. LCMS $(\mathrm{m} / \mathrm{z})$ : calculated $\left(\mathrm{M}^{+}+\mathrm{Na}\right)$ : 378 , found 378.31 .

5. Diethyl 2,6-dimethyl-4-(2-nitrophenyl)-1,4dihydropyridine-3,5-dicarboxylate (1e):

$\%$ Yield 75 ; m.p. $172-174{ }^{\circ} \mathrm{C}$.

IR $\nu_{\max } / \mathrm{cm}^{-1}: 3343(\mathrm{~N}-\mathrm{H}), 2888(\mathrm{C}-\mathrm{H}), 1702(\mathrm{C}=\mathrm{O})$, $1642(\mathrm{C}=\mathrm{O}), 1523(=\mathrm{C}-\mathrm{H}$ and $\operatorname{ring} \mathrm{C}=\mathrm{C}), 1485-1433$ $(\mathrm{C}=\mathrm{C}), 1369\left(\mathrm{CH}_{3}\right.$ in $\left.\mathrm{O}-\mathrm{CH}_{2}-\mathrm{CH}_{3}\right), 1346(\mathrm{C}-\mathrm{N}$ in $-\mathrm{N})$, 1298-1248 (C-O-C). ${ }^{1} \mathrm{H}$ NMR $\left(500.1 \mathrm{MHz} ; \mathrm{CDCl}_{3}\right), \delta$ : $1.193\left(3 \mathrm{H}, \mathrm{CH}_{3}\right), 2.466\left(3 \mathrm{H}, \mathrm{CH}_{3}\right), 2.42\left(2 \mathrm{H}, \mathrm{CH}_{2}\right)$, $5.09(1 \mathrm{H}, \mathrm{CH}), 5.84-7.27(4 \mathrm{H}, \mathrm{Ar}-\mathrm{H}), 6.46(1 \mathrm{H}, \mathrm{NH})$.

6. Diethyl 4-(2-bromophenyl)-2,6-dimethyl-1,4dihydropyridine-3,5-dicarboxylate (1f):

$\%$ Yield 65 ; m.p. $210-213{ }^{\circ} \mathrm{C}$.

${ }^{1} \mathrm{H} \mathrm{NMR}\left(500.1 \mathrm{MHz}, \mathrm{CDCl}_{3}\right), \delta: 1.05\left(3 \mathrm{H}, \mathrm{CH}_{3}\right), 2.12$ $\left(3 \mathrm{H}, \mathrm{CH}_{3}\right), 2.30\left(2 \mathrm{H}, \mathrm{CH}_{2}\right), 5.00(1 \mathrm{H}, \mathrm{CH}), 6.77-7.58$ $(4 \mathrm{H}, \mathrm{Ar}-\mathrm{H}), 5.82(1 \mathrm{H}, \mathrm{NH})$.

7.Diethyl 4-(4-hydroxyphenyl)-2,6-dimethyl-1,4dihydropyridine-3,5-dicarboxylate $(1 \mathrm{~g})$ :

$R_{f}: 0.60 ; \%$ Yield: $93 ;$ m.p. $241^{\circ} \mathrm{C}$.

IR spectrum $\left(\nu_{\max } / \mathrm{cm}^{-1}\right): 3344(\mathrm{~N}-\mathrm{H}), 2986(\mathrm{C}-\mathrm{H})$, $1792(\mathrm{C}=\mathrm{O}), 1716(\mathrm{C}=\mathrm{O}), 1540(=\mathrm{C}-\mathrm{H}$ and $\operatorname{ring} \mathrm{C}=\mathrm{C})$, $1218(\mathrm{C}-\mathrm{O}-\mathrm{C})$.

Mass $(\mathrm{m} / \mathrm{z})$ : calculated $\left(\mathrm{M}^{+}+\mathrm{Na}\right)$ : 368 , found 368.28 . UV (absorption): $400 \mathrm{~nm}$.

8.Diethyl-4-(4-bromophenyl)-2,6-dimethyl-1,4dihydropyridine-3,5-dicarboxylate $(1 \mathrm{~h})$ :

$R_{f}: 0.70 ; \%$ Yield: $60 ;$ m.p. $211^{\circ} \mathrm{C}$.

${ }^{1} \mathrm{H}$ NMR: $\left(500.1 \mathrm{MHz} ; \mathrm{CDCl}_{3} ; \mathrm{Me}_{4} \mathrm{Si}\right): 1.41(\mathrm{t}, 3 \mathrm{H}$, $\left.\mathrm{CH}_{3}\right), 2.32\left(\mathrm{~s}, 3 \mathrm{H}, \mathrm{CH}_{3}\right), 2.41\left(\mathrm{q}, 2 \mathrm{H}, \mathrm{CH}_{2}\right), 5.19$ (s, 1 $\mathrm{H}, \mathrm{CH}), 5.93-6.20$ (m, $4 \mathrm{H}, \mathrm{Ar}-\mathrm{H}), 4.41$ (s, $1 \mathrm{H}, \mathrm{NH})$.

9. Diethyl 2',6'-dimethyl-1',4'-dihydro-2,4'bipyridine-3',5'-dicarboxylate (1i):
IR $\left(\nu_{\max } / \mathrm{cm}^{-1}\right): 3100(\mathrm{~N}-\mathrm{H}), 2927(\mathrm{C}-\mathrm{H}), 1685$ $(\mathrm{C}=\mathrm{O}), 1632(\mathrm{C}=\mathrm{O}), 1504 \quad(=\mathrm{C}-\mathrm{H}$ and ring $\mathrm{C}=\mathrm{C})$, $1428(\mathrm{C}=\mathrm{C}), 1377\left(\mathrm{CH}_{3}\right.$ in $\left.\mathrm{O}-\mathrm{CH}_{2}-\mathrm{CH}_{3}\right), 1300(\mathrm{C}-\mathrm{N}$ in $\left.\mathrm{C}_{A r}-\mathrm{N}\right) ; 1266$ (C-O-C). ${ }^{1} \mathrm{H}$ NMR (500.1 MHz, $\mathrm{CDCl}_{3}$; $\left.\mathrm{Me}_{4} \mathrm{Si}\right), 1.12\left(3 \mathrm{H}, \mathrm{CH}_{3}\right), 2.32\left(2 \mathrm{H}, \mathrm{CH}_{2}\right), 2.48(3 \mathrm{H}$, $\left.\mathrm{CH}_{3}\right), 4.15(1 \mathrm{H}, \mathrm{CH}), 4.721(1 \mathrm{H}, \mathrm{NH}), 6.50-8.71$ (4 $\mathrm{H}, \mathrm{Ar}-\mathrm{H})$. MS $(\mathrm{m} / \mathrm{z})$ : calculated $\left(\mathrm{M}^{+}+\mathrm{K}\right)$ : 369 , found 368.28 .

10. Diethyl 2,6-dimethyl-4-(3-nitrophenyl)-1,4dihydropyridine-3,5-dicarboxylate $(\mathbf{1 j})$ :

IR $\left(\nu_{\max } / \mathrm{cm}^{-1}\right): 3343(\mathrm{~N}-\mathrm{H}), 2888(\mathrm{C}-\mathrm{H}), 1702$ $(\mathrm{C}=\mathrm{O}), 1642(\mathrm{C}=\mathrm{O}), 1523(=\mathrm{C}-\mathrm{H}$ and $\operatorname{ring} \mathrm{C}=\mathrm{C}), 1485-$ $1433(\mathrm{C}=\mathrm{C}), 1369\left(\mathrm{CH}_{3}\right.$ in $\left.\mathrm{O}-\mathrm{CH}_{2}-\mathrm{CH}_{3}\right), 1346(\mathrm{C}-\mathrm{N}$ in -N), 1298-1248 (C-O-C). ${ }^{1} \mathrm{H}$ NMR (500.1 MHz, $\mathrm{CDCl}_{3}$, $\left.\mathrm{Me}_{4} \mathrm{Si}\right), 1.193\left(3 \mathrm{H}, \mathrm{CH}_{3}\right), 2.466\left(3 \mathrm{H}, \mathrm{CH}_{3}\right), 2.42(2 \mathrm{H}$, $\left.\mathrm{CH}_{2}\right), 5.09(1 \mathrm{H}, \mathrm{CH}), 5.84-7.27(4 \mathrm{H}, \mathrm{Ar}-\mathrm{H}), 6.46(1 \mathrm{H}$, $\mathrm{NH})$.

11. Diethyl 4-(furan-3-yl)-2,6-dimethyl-1,4dihydropyridine-3,5-dicarboxylate (1k):

IR $\left(\nu_{\max } / \mathrm{cm}^{-1}\right): 3344(\mathrm{~N}-\mathrm{H}), 2960(\mathrm{C}-\mathrm{H}), 1697$ $(\mathrm{C}=\mathrm{O}), 1644 \quad(\mathrm{C}=\mathrm{O}), 1539 \quad(=\mathrm{C}-\mathrm{H}$ and ring $\mathrm{C}=\mathrm{C})$, $1475(\mathrm{C}=\mathrm{C}), 1369\left(\mathrm{CH}_{3}\right.$ in $\left.\mathrm{O}-\mathrm{CH}_{2}-\mathrm{CH}_{3}\right), 1320(\mathrm{C}-\mathrm{N}$ in $\left.\mathrm{C}_{A r}-\mathrm{N}\right), 1296$ (C-O-C). ${ }^{1} \mathrm{H}$ NMR (500.1 MHz, $\mathrm{CDCl}_{3}$, $\left.\mathrm{Me}_{4} \mathrm{Si}\right): 1.23\left(3 \mathrm{H}, \mathrm{CH}_{3}\right), 2.32\left(3 \mathrm{H}, \mathrm{CH}_{3}\right), 2.18(2 \mathrm{H}$, $\left.\mathrm{CH}_{2}\right), 5.19(1 \mathrm{H}, \mathrm{CH}), 5.94-6.20(3 \mathrm{H}, \mathrm{Ar}-\mathrm{H}), 4.30(1 \mathrm{H}$, $\mathrm{NH})$.

\subsection{Spectral representation for representative compound - diethyl 2,6-dimethyl-4-(4-nitrophenyl)-1,4-dihydro- pyridine-3,5-dicarboxylate (1a)}

Diethyl 2,6-dimethyl-4-(4-nitrophenyl)-1,4-dihydropyridine-3,5-dicarboxylate (1a) was characterized by ${ }^{1}$ H NMR (Fig. 7), LC-MS (Fig. 8), FTIR (Fig. 9), and ${ }^{13}$ C DEPT NMR (Fig. 10) spectral analysis.

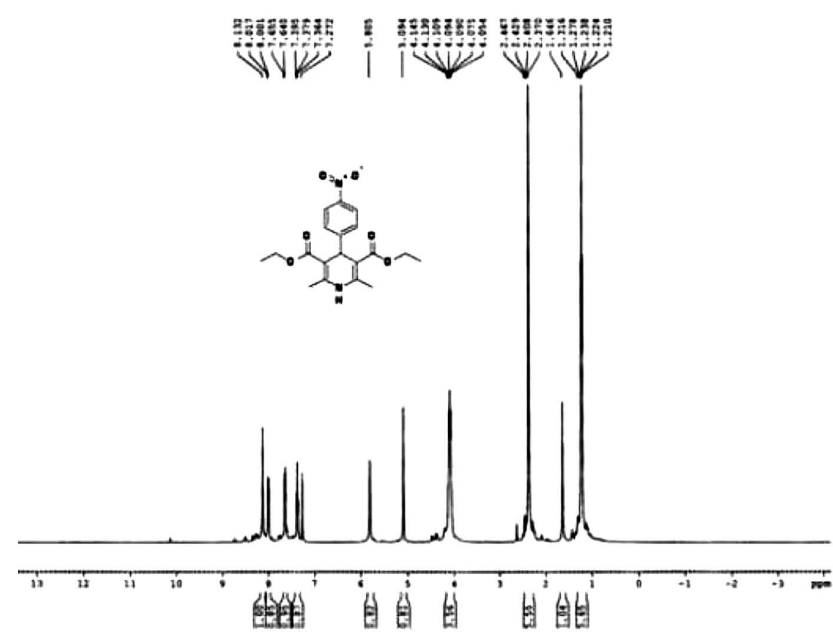

Fig. 7. ${ }^{1} \mathrm{H}$ MNR spectrum of $1 \mathrm{a}$.

Diethyl 2,6-dimethyl-4-(4-nitrophenyl)-1,4-dihydropyridine-3,5-dicarboxylate (1a) was additionally characterized by ${ }^{13} \mathrm{C}$ distortionless enhancement by polarization transfer (DEPT). 


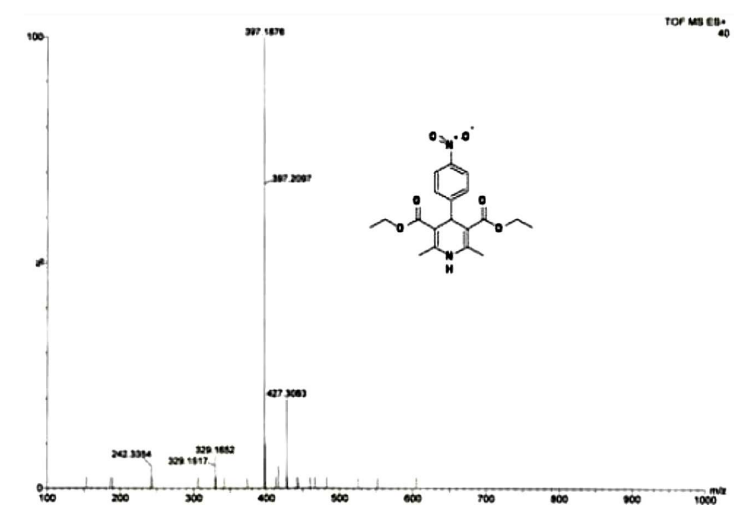

Fig. 8. LC-MS spectrum of 1a.

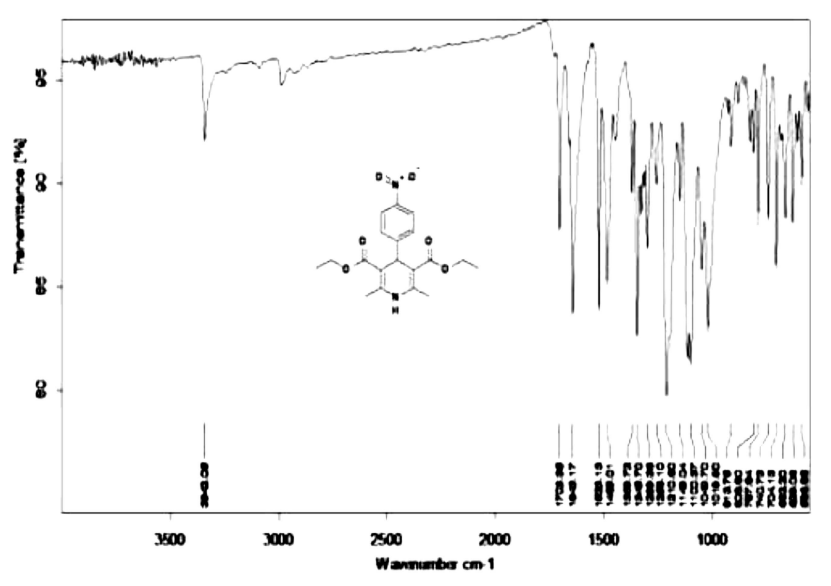

Fig. 9. FTIR spectrum of $1 \mathrm{a}$.

The DEPT spectral analysis is a useful spectroscopic method for determination of different types of primary, secondary and tertiary carbon atoms. The DEPT spectroscopic method gives different signals for $\mathrm{CH}, \mathrm{CH}_{2}$ and $\mathrm{CH}_{3}$ groups by variation of the selection angle parameter. The DEPT spectrum of 1a (Fig. 8) shows the signals for $\mathrm{CH}$ and $\mathrm{CH}_{3}$ in an upward direction while the signal of $\mathrm{CH}_{2}$ in the opposite direction. So the structure is confirmed by ${ }^{13} \mathrm{C}$ DEPT spectra.

The spectral data confirm the structure of synthesized compounds. The optimization study reveals that the $\mathrm{CuO}$ nanoparticle as an effective catalyst for synthesis of 1,4-dihydropyridine derivative, i.e. 2,6-dimethyl-4-(4nitrophenyl)-1,4-dihydropyridine-3,5-dicarboxylate (1a). Moreover, the proposed protocol is microwave assisted, environmental benign, proficient, and fast method for synthesis of bio-inspired dihydropyridine derivative.

\section{Conclusion}

A competent, simplistic and economical method for the synthesis of functionalized dihydropyridine derivatives was reported here, using $\mathrm{CuO}$ nanoflakes as a catalyst in an ethanol/water mixture green media. The products were obtained in remarkable yields and the reaction

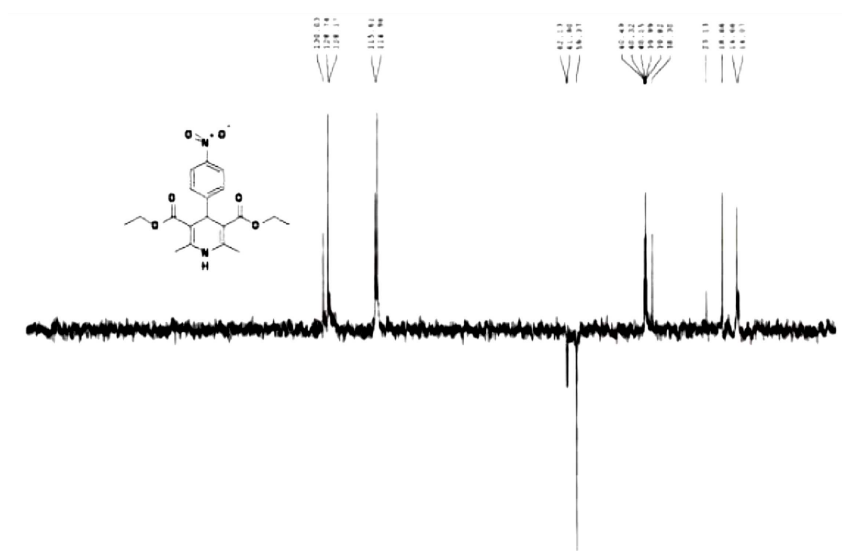

Fig. 10. ${ }^{13} \mathrm{C}$ DEPT spectrum of $1 \mathrm{a}$.

times were considerably low. The present protocol represents an easy and a remarkable method for the threecomponent reactions to synthesize some dihydropyridine derivatives using unique nanoscale materials. Moreover, the reusability of this $\mathrm{CuO}$ nanocatalyst is one of its important character and also this potency inspired us to use it for commercial and industrial organic synthesis applications.

\section{References}

[1] M.B. Gawande, R. Luque, R. Zboril, ChemCatChem 6, 3312 (2014).

[2] M.B. Gawande, P.S. Brancoa, R.S. Varma, Chem. Soc. Rev. 42, 3371 (2013).

[3] D. Wang, D. Astruc, Chem. Rev. 114, 6949 (2014).

[4] Q.M. Kainz, R. Linhardt, R.N. Grass, Adv. Funct. Mater. 24, 2020 (2014).

[5] S.N. Shelke, S.R. Bankar, G.R. Mhaske, S.S. Kadam, D.K. Murade, ACS Sustain. Catal. Eng. 2, 1699 (2014).

[6] S. Sá, M.B. Gawande, A. Velhinho, J.P. Veiga, N. Bundaleski, Green Chem. 16, 3494 (2014).

[7] M.B. Gawande, I. Rathi, D. Nogueira, Green Chem. 15, 1895 (2013).

[8] M. Shokouhimehr, Y. Piao, J. Kim, Y. Jang, T. Hyeon, Angew. Chem. Int. Ed. 46, 7039 (2007).

[9] F. Shirini, M. Abedini, J. Nanosci. Nanotechnol. 13, 4838 (2013).

[10] H. Montazeri, A. Amani, H.R. Shahverdi, E. Haratifar, A.R. Shahverdi, J. Nanostruct Chem. 3, 25 (2013).

[11] P. Arumugam, P.T. Perumal, Ind. J. Chem. Sect. B Org. Incl. Med. 47, 1084 (2008).

[12] A.W. Bauer, W.M.M. Kirby, J.C.T. Sherris, M. Turck, Am. J. Clin. Pathol. 45, 493 (1966).

[13] R.A. Janis, D.J. Triggle, J. Med. Chem. 26, 775 (1983).

[14] R.H. Boecker, F.P. Guengerich, J. Med. Chem. 29, 1596 (1986). 
[15] M.F. Gordeev, D.V. Patel, E.M. Gordon, J. Org. Chem. 61, 924 (1996).

[16] C.E. Sunkel, M. Fau de Casa-Juana, L. Santos, A.G. Garcia, C.R. Artalejo, M. Villarroya, J. Cillero, J. Med. Chem. 35, 2407 (1992).

[17] D. Vo, W.C. Matowe, M. Ramesh, N. Iqbal, M.W. Wolowyk, S.E. Howlett, E.E. Knaus, J. Med. Chem. 38, 2851 (1995).

[18] S.A. Sadeek, W.A. Zordok, M.S. El-Attar, M.S. Ibrahim, Bull. Chem. Soc. Ethiopia 29, 75 (2015).

[19] Z. Luo, F. He, J. Luo, W. Zhang, Am. J. Life Sci. 3, 1 (2015).

[20] V. Klusa, Drugs Fut. 20, 135 (1995).

[21] T. Godfraind, R. Miller, M. Wibo, Pharmacol. Rev. 38, 321 (1986).

[22] A. Sausins, G. Duburs, Heterocycles 27, 269 (1988).

[23] A.C. Gaudio, A. Korolkovas, Y. Takahata, J. Pharm. Sci. 83, 1110 (1994).

[24] K. Cooper, M.J. Fray, M.J. Parry, K. Richardson, J. Steele, J. Med. Chem. 35, 3115 (1992).

[25] A. Agarwal, P.M.S. Chauhan, Tetrahedron Lett. 46 , 1345 (2005)

[26] L. Ohberg, J. Westman, Synlett 8, 1296 (2001).

[27] J.G. Breitenbucher, G. Figliozzi, Tetrahedron Lett. 41, 4311 (2000).

[28] J.C. Liang, J.L. Yeh, C.S. Wang, S.F. Liou, C.H. Tasi, I. Chen, J. Bioorg. Med. Chem. Lett. 10, 719 (2002).

[29] R. Miri, H. Niknahad, G. Vesal, A. Shafiee, Farmaco 57, 123 (2002).

[30] A. Dondoni, A. Massi, E. Minghini, S. Sabbatini, V. Bertoasi, J. Org. Chem. 68, 6172 (2002).

[31] A. Dondoni, A. Massi, E. Minghini, V. Bertoasi, Tetrahedron 60, 2311 (2004).
[32] S.J. Ji, Z.Q. Jiang, J. Lu, T.P. Loh, Synlett 5, 831 (2004).

[33] M. Maheswara, V. Siddaiah, G.L. Damu, C. Venkata Rao, Arkivoc 2, 201 (2006).

[34] M.A. Chari, K. Syamasundar, Catal. Commun. 6, $624(2005)$.

[35] S. Ko, M.N.V. Sastry, C. Lin, C.F. Yao, Tetrahedron Lett. 46, 5771 (2005).

[36] A. Kumar, R.A. Maurya, Tetrahedron Lett. 48, 3887 (2007).

[37] L.M. Wang, J. Sheng, L. Zhang, J.W. Han, Z. Fan, H. Tian, C.T. Qian, Tetrahedron 61, 1539 (2005).

[38] N. Tewari, N. Dwivedi, R.P. Tripathi, Tetrahedron Lett. 45, 9011 (2004).

[39] A. Kumar, R.A. Maurya, Tetrahedron 63, 1946 (2007).

[40] S.R. Cherkupally, R. Mekalan, Chem. Pharm. Bull. 56, 1002 (2008).

[41] H. Adibi, H.A. Samimi, M. Beygzadeh, Catal. Commun. 8, 2119 (2007).

[42] G. Sabitha, G.S.K. Reddy, C.S. Reddy, J.S. Yadav, Tetrahedron Lett. 44, 4129 (2003).

[43] S.B. Sapkal, K.F. Shelke, B.B. Shingate, M.S. Shingare, Tetrahedron Lett. 50, 1754 (2009).

[44] A. Maleki, Tetrahedron 68, 7827 (2012).

[45] A. Maleki, Tetrahedron Lett. 54, 2055 (2013).

[46] A. Maleki, N. Ghamari, M. Kamalzare, RSC Adv. 4 9416 (2014).

[47] A. Maleki, M. Kamalzare, Catal. Commun. 53, 67 (2014).

[48] A. Maleki, R. Rahimi, S. Maleki, N. Hamidi, $R S C$ Adv. 4, 29765 (2014). 